\title{
Long-term hazards of neonatal blue-light phototherapy
}

\author{
J. Oláh, ${ }^{1}$ E. Tóth-Molnár, ${ }^{2}$ L. Kemény ${ }^{1,3}$ and Z. Csoma $^{1}$ \\ ${ }^{1}$ Department of Dermatology and Allergology, ${ }^{2}$ Department of Ophthalmology and ${ }^{3}$ Dermatological Research Group of the Hungarian Academy of Sciences, Univer- \\ sity of Szeged, Szeged, Hungary
}

\section{Summary}

\section{Correspondence \\ Zsanett Csoma. \\ E-mail: csomazs@mail.derma.szote.u-szeged.hu}

\section{Accepted for publication}

16 March 2013

\section{Funding sources}

None.

\section{Conflicts of interest}

None declared.

DOI $10.1111 /$ bjd.12335
Blue-light phototherapy has been an essential therapeutic tool in the management of neonatal jaundice for decades. Rarely, it is accompanied by acute dermatological and systemic side-effects, but fortunately these are reversible and can be adequately and promptly treated in routine neonatal practice. In contrast, much less is known about the potential long-term side-effects of neonatal blue-light phototherapy (NBLP). Many of the data that are currently available on how NBLP influences melanocytic naevus (MN) development are controversial. The results of recent well-designed epidemiological surveys suggest that NBLP could well be a risk factor for MN formation, and highlight the need for additional in vivo and in vitro studies. NBLP is at present the mainstay of treatment for neonatal jaundice, but in the future greater consideration should be given to its long-term sideeffects when phototherapy is indicated. It is relevant to emphasize the importance of appropriately restricted and adequate clinical guidelines, and strict monitoring of the management of hyperbilirubinaemia, in order to avoid the unnecessary overtreatment of newborn infants.

\section{What's already known about this topic?}

- Neonatal blue-light phototherapy (NBLP) is at present the mainstay of treatment for neonatal jaundice, and has proved to be an essential and generally safe modality for the prevention of bilirubin encephalopathy.

- Many of the data that are currently available on how NBLP influences melanocytic naevus development are controversial.

\section{What does this study add?}

- This review article assesses the available dermatological and ophthalmological evidence regarding the possible effects of NBLP on melanocytic naevus development.
Neonatal blue-light phototherapy (NBLP) has been an essential therapeutic tool in the management of neonatal jaundice for decades (Fig. 1). Millions of full-term and preterm infants have received this very effective, simple and safe therapy to reduce the concentration of serum bilirubin. NBLP may be accompanied by acute dermatological and systemic sideeffects, but fortunately these are reversible, and can be adequately and promptly treated in routine neonatal practice. Thanks to the restricted treatment protocols, the prevalence of short-term adverse events is currently very low. These minor and transient complications include skin burns, erythematous skin rash, purpuric and bullous eruptions, retinal damage, thermoregulatory instability, irritability, loose stools, dehydra- tion, feeding difficulties and the 'bronze-baby' syndrome. ${ }^{1-3}$ Chen et al. ${ }^{4}$ have reported significant alterations in circadian gene expression and melatonin secretion among neonates receiving NBLP, resulting in changes in circadian rhythm and behaviour.

In contrast, much less is known about the potential longterm side-effects of NBLP. It should be recalled that the wavelengths of blue light (BL) and ultraviolet (UV) radiation are adjacent, and their biological effects might therefore partially overlap. Moreover, the traditional and widely used BL lamps emit a minor UVA radiation component in addition to the therapeutic blue wavelengths (Fig. 2). ${ }^{5}$ The erythema and tanning of the newborn skin observed after phototherapy might 


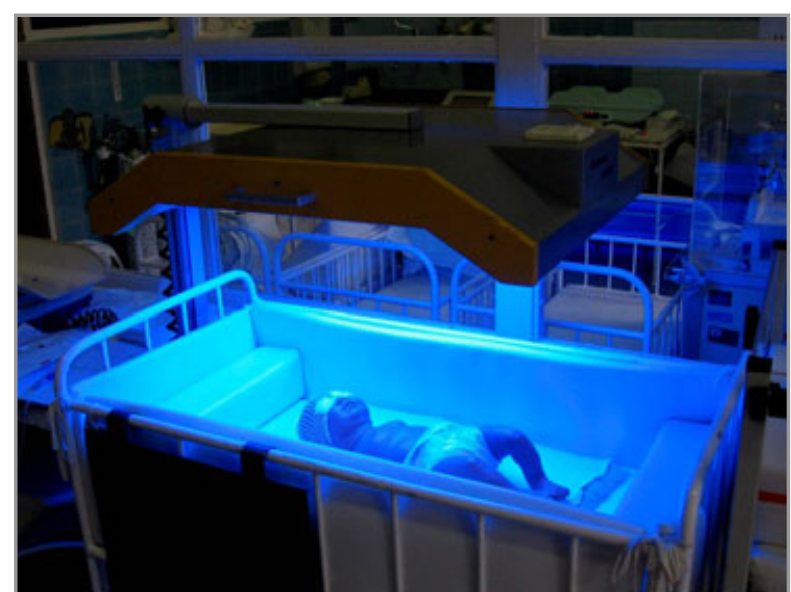

Fig 1. Neonatal blue-light phototherapy.

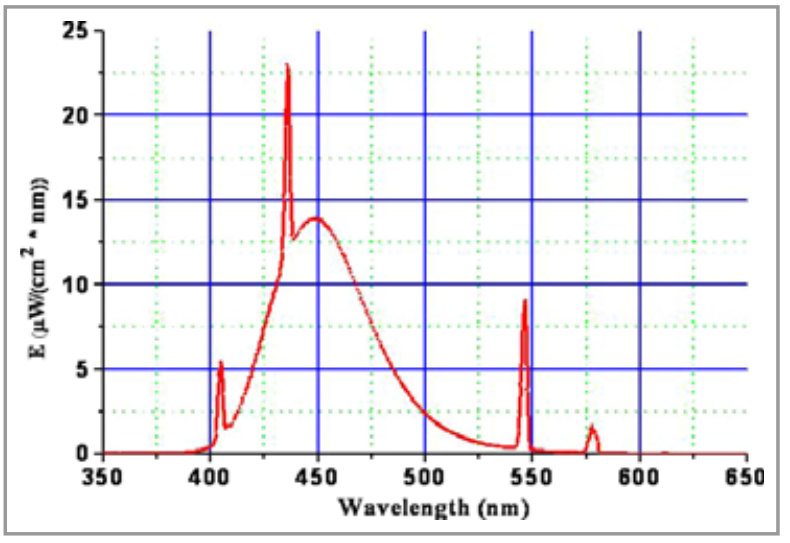

Fig 2. Emission spectrum of the commonly used, traditional bluelight lamp with Plexiglas cover between the fluorescent bulbs and the infant. According to measurements, the emission spectrum of the blue-light lamps used in Hungary is between $370 \mathrm{~nm}$ and $600 \mathrm{~nm}$, with a maximum at $450 \mathrm{~nm}$. Approximately $0.3 \%$ of the output comprises ultraviolet (UV) A radiation. Our measurements were made with a QE6500 spectrophotometer (Ocean Optics, Dunedin, FL, U.S.A.) at the exact position of the infants, and suggest that a minor component of UVA radiation can transmit through the Plexiglas cover.

be due to this small amount of UV irradiation. ${ }^{6}$ UV irradiation has profound immunosuppressive and immunomodulatory effects; it induces melanocyte proliferation and plays an important role in naevogenesis. Visible light has similar physiological effects to those of UV radiation, as it induces the production of reactive oxygen species and increases the release of proinflammatory cytokines. ${ }^{7}$ Visible light can penetrate deeper into the skin than can UV irradiation, and may possibly give rise to significant biological effects on the epidermal and dermal cells. Similarly to UV radiation, BL may exert cytotoxic effects, inducing significant oxidative stress, DNA damage and sister chromatid exchange, and may also influence the immune system. ${ }^{8-11}$ Aspberg et al. ${ }^{10}$ reported a possible association between neonatal phototherapy and asthma in hospitalized children, as a long-term consequence of the influence of phototherapy on the immature immune system.

Immediate adverse effects of NBLP on the neonatal eye are well known in clinical practice: accidental exposure can cause periocular skin erythema, bacterial infection and photokeratitis. This latter damage of the corneal epithelium is a consequence of direct exposure of the anterior surface of the eye. Retinal photodamage was investigated in primates in one study by Messner et al. ${ }^{12}$ Newborn monkeys were continuously exposed to high-intensity fluorescent light. The animals could open and close their eyelids as desired during the exposure. The newborn primate retina was damaged in a progressive manner. Morphological retinal damage could be proven as early as after $12 \mathrm{~h}$ of exposure (the shortest interval examined). With increasing periods of exposure, the retinal damage became more extensive. ${ }^{12}$

The long-term effects have been only poorly investigated. In a group of 4-year-old children who participated in NBLP, Dobson et al. ${ }^{13}$ did not observe any ophthalmological changes, including a lack of electroretinographic alterations. The possibility of an association between ocular and cutaneous melanocytes follows from the fact that uveal melanocytes and melanocytes of the conjunctiva and the skin all originate in the neural crest and migrate to their respective sites during embryological development. These morphologically similar dendritic melanocytes, which reside in the skin, conjunctiva, iris, ciliary body and choroid, may give rise to naevi or melanomas at these respective sites. ${ }^{14,15}$ As far as we are aware, our 2011 article provided the first literature survey of ocular pigmented alterations in patients treated with NBLP during the early neonatal period. ${ }^{16}$

As some attempts have been made in the past few years to investigate the possible long-term impacts of NBLP concerning the development of melanocytic naevus $(\mathrm{MN})$, in this review article we set out to assess the available dermatological and ophthalmological evidence.

\section{Neonatal blue-light phototherapy and cutaneous melanocytic naevus}

In the first study in the literature, Bauer et al. ${ }^{17}$ conducted a cross-sectional study on 1812 white children aged 2-7 years. A notably high number $(n=333)$ of the children had received NBLP. It was concluded from both bivariate and multivariate analyses that NBLP was not associated with an increased risk of the development of $\mathrm{MN}$; the untreated and the treated children exhibited the same median number of MNs. ${ }^{17}$

In a prospective case-control study, Matichard et al. ${ }^{18}$ found that intensive NBLP was a strong risk factor for the development of naevi in childhood. They compared the naevus count in two groups of 8-9-year-old children, one group consisting of 18 subjects treated with intensive NBLP in the first few days of life, and the control group consisting of 40 untreated children. The number of naevi measuring $\geq 2 \mathrm{~mm}$ was 
significantly higher in the exposed group. When the analysis was limited to naevi measuring $2-5 \mathrm{~mm}$, the difference proved to be even more significant. Conversely, the association between NBLP and the total naevus count was not significant for naevi $<2 \mathrm{~mm}$ or $>5 \mathrm{~mm}$ in size. However, the relatively small groups limited the power of the results. ${ }^{18}$

In the multicentre study by Mahé et al. ${ }^{19}$ on a homogeneous population of 9-year-old children, NBLP was not associated with an increase in the number of naevi, irrespective of their location or size. In total, 180 of the 828 children studied had received phototherapy. Naevi measuring $<2 \mathrm{~mm}, \quad 2-5 \mathrm{~mm}$ and $>5 \mathrm{~mm}$ were counted separately, and the evaluation was blinded for the history of NBLP.

Our own initial study revealed a significantly higher prevalence of clinically atypical MNs (CAMNs) among schoolchildren aged 14-18 years who had received NBLP. In total, 747 schoolchildren were investigated to determine the prevalence of common MN (CMN) and CAMN. Data were recorded with regard to the neonatal history, such as prematurity, neonatal jaundice and NBLP; $44 \cdot 6 \%$ of the children had received NBLP. The prevalence of dysplastic naevi was $19 \cdot 1 \%$ in the untreated group and $25 \cdot 2 \%$ in the treated group. NBLP resulted in a relative risk of 1.32 for the development of CAMN. The prevalence of $\mathrm{CMN}$ was quite similar in the treated and the untreated children, but the exposed subjects were somewhat more likely to exhibit multiple moles. ${ }^{20}$

It is worthy of mention that NBLP has been utilized for the treatment of neonatal jaundice in Hungary since 1968. We therefore considered the question of whether there was a difference in the prevalence of CAMN between those born before or after the introduction of NBLP. In 2006, in the course of an employment screening programme, 618 healthy, unselected subjects underwent whole-body skin examinations. We found that the prevalence of CAMN was significantly higher among those born in or after 1968 than among those born before 1968. The prevalence of CAMN in the two groups was $36.3 \%$ and $21 \cdot 2 \%$, respectively. ${ }^{21}$

Nevertheless, it is important to bear in mind the possibility that the increasing prevalence of MN among white populations in recent decades might be explained in part by the increased UV exposure due to climatic changes, the depletion of the protective ozone layer and the changes in sunbathing habits and sun-protection methods.

The striking results mentioned above led us to investigate the impact of NBLP on MN development in a more homogeneous population, where the role of environmental factors appears to be more similar. We examined monozygotic and dizygotic twins, where one of the twins had received phototherapy for neonatal jaundice, whereas the other had not. Fifty-eight pairs of twins (15 monozygotic and 43 dizygotic) and one set of triplets, of white origin, aged 3-30 years, were included in the study. ${ }^{16}$ Univariate analysis revealed that NBLP was associated with a significantly higher prevalence of both CMN and CAMN in the examined twin pairs (Fig. 3). When the analysis was focused separately on the monozygotic and dizygotic twin pairs, a statistically significant difference in the

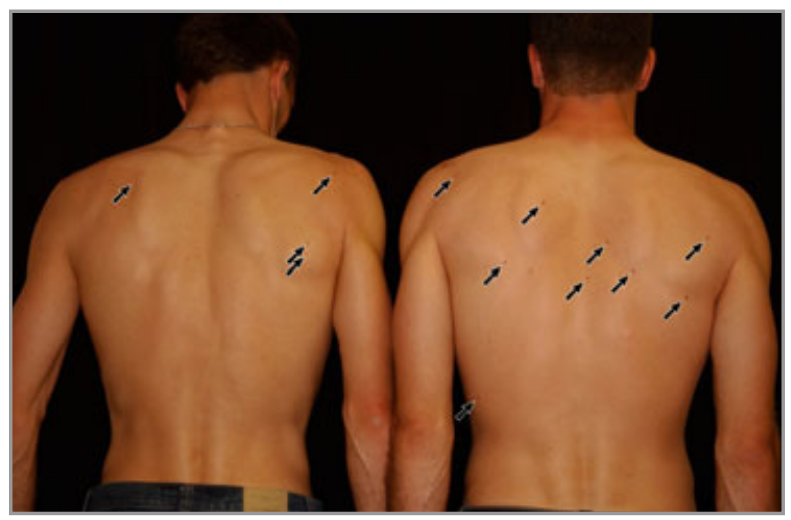

Fig 3. Difference in the number of melanocytic naevi in a monozygotic twin pair. The twin on the right-hand side, who received blue-light phototherapy, demonstrates a significantly higher naevus count than that of the sibling on the left-hand side, who did not receive neonatal phototherapy.

number of naevi was still observed between the exposed and nonexposed subjects in the case of the monozygotic twins. For the dizygotic twin pairs, the number of CMNs and the overall number of MNs differed in a statistically significant manner between the treated and untreated twin members. Multivariate linear regression analysis demonstrated that the number of MNs was also significantly and independently associated with a history of NBLP. A standardized questionnaire was used to assess the data relating to constitutional or sun exposure and other variables. These factors proved to be very consistent in the examined monozygotic twin pairs. The phenotypic characteristics of the dizygotic twins did differ to some extent, but the environmental impacts were very similar until adulthood. ${ }^{16}$

Various epidemiological data indicate that the presence of large numbers of CMNs and CAMNs is the most important independent phenotypic risk factor for the development of malignant melanoma in fair-skinned populations, and our results therefore raise the question of whether NBLP could also be a risk factor for melanoma. A preliminary case-control study suggested that there was no significant risk of the development of childhood malignant melanoma after NBLP. ${ }^{22}$ Melanoma was not observed either in the NBLP-exposed subjects or in the control groups in our surveys, but the follow-up period was relatively short.

\section{Neonatal blue-light phototherapy and the neonatal eye}

It has long been known that BL can induce retinal photoreceptor degeneration in rats and in mammals. ${ }^{23,24}$ Experimental results outlining the potentially retinotoxic effect of BL continue to accumulate. In general, the outer photoreceptor segments are the structures that are first injured, and this is followed by damage to the retinal pigment epithelial cells. ${ }^{25}$ Widespread apoptotic cell death has been demonstrated in the albino rat retina after $\mathrm{BL}$ illumination. ${ }^{26} \mathrm{~A}$ very similar effect 


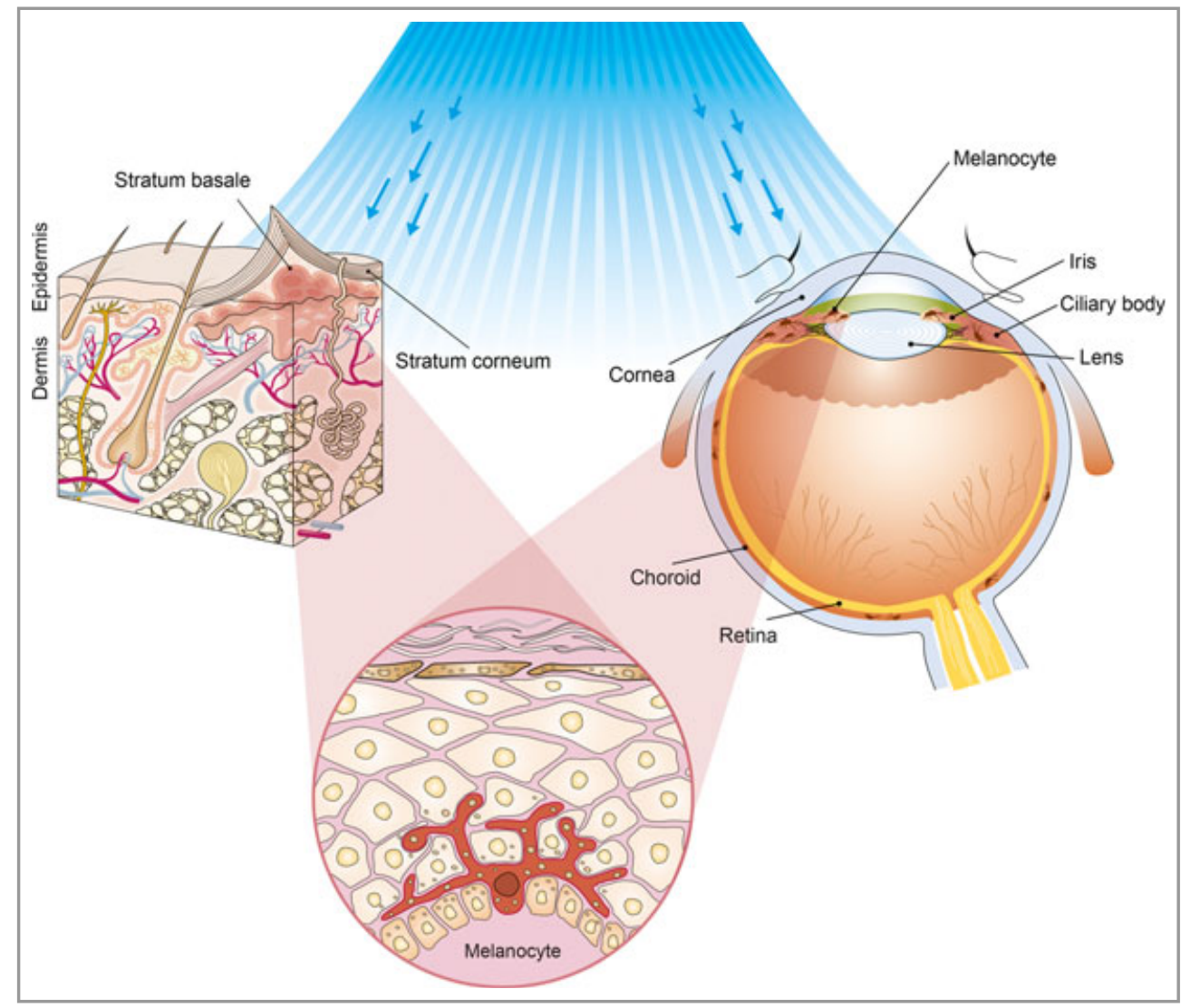

Fig 4. Blue-light exposure of cutaneous and uveal melanocytes during phototherapy. Ocular melanocytes consist of two different cell types: conjunctival and uveal melanocytes. Uveal melanocytes reside in the middle layer of the eyeball: in the iris, the ciliary body and the choroid. Conjunctival melanocytes are located in the conjunctiva (thin layers of epithelium and underlying connective tissue covering the anterior surface of the sclera and the posterior surface of the eyelids).

observed in BL-irradiated, cultured human retinal pigment epithelial cells could be prevented through use of a blueblocking filter. $^{27-29}$ These effects might be due to BL having a considerable capacity to remove electrons from molecules, resulting in reactive oxygen species. This photoelectric effect in the $400-500 \mathrm{~nm}$ interval has its peak at $440 \mathrm{~nm}$ and is referred to as the BL hazard.

There have been previous surveys of the possibility of exposure to BL eliciting the malignant transformation of uveal melanocytes (Fig. 4). Manning et al. ${ }^{30}$ found that long-term exposure to fluorescent BL resulted in the development of uveal epithelioid melanoma in rats. In another experimental animal model, Di Cesare et al. ${ }^{31}$ observed that exposure to BL led to a significantly higher rate of proliferation of human uveal melanoma cells relative to the unexposed, control group. The in vitro model utilized by Marshall et al. ${ }^{32}$ revealed that exposure to BL induced a significant increase in the rate of proliferation of four different human uveal melanoma cell lines.

There are a number of factors that may influence the extent to which the retinal pigment epithelial cells and photoreceptors are damaged. The spectral composition, duration, intermittence and intensity of the radiation, and the light transmittance characteristics of the given structures, besides interindividual variations, can all influence the degree of biological damage. ${ }^{33}$

The amount of radiation reaching different ocular structures additionally depends on the transmission profile of the ocular media. The cornea effectively filters UV radiation below $295 \mathrm{~nm}$ and transmits most of the UVA and UVB and visible spectrum throughout life, with merely a relatively minor reduction in percentage transmission in the elderly. ${ }^{34}$ Transmittance increases rapidly above $300 \mathrm{~nm}$, reaching $>90 \%$ above $500 \mathrm{~nm}$. The characteristics of the light transmission of the crystalline lens are highly age dependent. Whereas in neonates the lens transmits $>90 \%$ of the light with wavelengths below $450 \mathrm{~nm}$, this declines to $<20 \%$ by 80 years of age due to the progressive accumulation of lenticular chromatophores. $^{35-37}$

Our twin study demonstrated that NBLP was associated with a substantially higher prevalence of benign ocular pigmented lesions. ${ }^{16}$ The number of melanocytic lesions of the iris in our study proved to be independent of age. This can be explained by the time course of iris pigmentation: the melanin concentration peaks during early childhood, thereafter usually remaining constant throughout life unless affected by certain 
ocular disorders, which can lead to hypopigmentation or hyperpigmentation. ${ }^{38,39}$

In contrast with cutaneous MN and cutaneous melanoma, only inconsistent data are available regarding both the factors that may influence the formation of uveal pigmented lesions and the role of benign pigmented ocular lesions as melanoma risk indicators. Nordlund et al. and Albert et al. ${ }^{40,41}$ reported that the total number of iris naevi was significantly higher in patients with cutaneous melanoma than in controls. Weis et al. ${ }^{15}$ recently published a meta-analysis that supports the correlation of uveal melanoma with CAMNs, CMNs and iris naevi. We earlier found a substantially higher prevalence of ocular pigmented findings (conjunctival and uveal naevi) in patients with dysplastic naevus syndrome compared with dysplastic naevus-free volunteers. ${ }^{42}$ In another study, we observed an increased rate of cutaneous dysplastic naevi in patients with uveal melanoma or with cutaneous melanoma. The relative risks of uveal melanoma and cutaneous melanoma in patients bearing atypical moles proved to be 4.36 and $4 \cdot 22$, respectively. ${ }^{43}$ Our investigations have additionally revealed that the presence of cutaneous dysplastic naevi in patients with uveal melanoma is associated with higher proportions of the prognostically worst forms of uveal melanoma. ${ }^{44}$

The ocular media of neonates are highly transmissive relative to those of adults, especially in the blue and UV regions of the spectrum. In the course of NBLP, the eyes are routinely patched or shielded with phototherapy hoods in order to exclude ocular exposure. ${ }^{45,46}$ Such protective measurements can exclude $>90 \%$ of the light emitted by phototherapy sources, but accidental exposure may occur. ${ }^{47,48}$ In one study, the eye shields were displaced in over half of all observations. ${ }^{49}$ On the other hand, precise patching of an infant's eye may be of secondary importance in comparison with more immediate and potentially life-preserving interventions. Although the potential hazard of BL is alleviated by the fact that neonates tend to keep their eyes shut in bright light, it is known that light in the visible spectrum penetrates the skin. The level of BL transmission through the closed eyelids of infants cannot be assessed with accuracy. The peak transmission is at the red end of the spectrum, and approximately $14 \%$ of the light at this wavelength can be transmitted in the adult, and $21 \%$ in the preterm infant. ${ }^{50,51}$ It has been estimated that at both ages around 3\% of the light with wavelengths below $580 \mathrm{~nm}$ can pass through the eyelid. Light does not enter the eye exclusively through the pupil, as the sclera and choroid also transmit about $14 \%$ of the light falling on them, but it does predominantly at the red end of the spectrum. With regard to the light transmissibility profile of the neonatal eye, which allows the penetration of an appreciable amount of potentially harmful light into the eye, further studies are needed to clarify the possible long-term effects of neonatal BL exposure on the melanocytic proliferation of the uveal tract. ${ }^{37,52}$ In the event of unavoidable phototherapy treatment, alternative methods of eye protection should be used in order to minimize accidental BL exposure of the extremely vulnerable neonatal eye.

\section{Discussion}

As with many of the findings of pivotal importance in medical science, the recognition of the beneficial effect of natural light on neonatal jaundice was serendipitous. ${ }^{53}$ Phototherapy is now widely and successfully applied to the treatment of neonatal jaundice in order to reduce the plasma bilirubin concentration and hence to prevent the need for exchange transfusion. Bilirubin absorbs light most strongly in the blue region of the spectrum $(425-475 \mathrm{~nm})$. The absorption of light by dermal and subcutaneous bilirubin induces various photochemical reactions, and the toxic, native, unconjugated bilirubin is converted to less toxic, water-soluble photoisomers, which are excreted in the bile and urine without requiring conjugation. Commonly used phototherapy units include daylight, cool-white, green, blue or 'special blue' fluorescent tubes. Other units involve tungsten-halogen lamps, highintensity gallium nitride light-emitting diodes or fibre-optic systems. ${ }^{54}$ When the total serum bilirubin level approaches the range in which intensive phototherapy is recommended, it is particularly important to use lamps with the clinically most effective blue emission spectrum. The American Academy of Pediatrics currently recommends the application of special blue fluorescent lamps or light-emitting diode lights. ${ }^{55}$

A large number of term and preterm infants are treated with phototherapy worldwide. The considerable variations between international and national guidelines often result in the overuse of phototherapy. ${ }^{56}$ It is relevant to clarify the potential prompt and long-term hazards of this widely accepted and used therapeutic modality as there is a growing body of evidence that phototherapy can potentially exert various effects on neonates, because of the unique physiological and pharmacological characteristics of this population.

In this review article we have attempted, as far as possible, to survey the results of previous clinical studies relating to the effects of NBLP on naevus development. In four of the six studies, an association was found between NBLP and naevus development, ${ }^{16,18,20,21}$ whereas the other two studies did not confirm this. ${ }^{17,19}$ It is rather difficult to compare the outcomes of these six surveys for several reasons. The sizes of the study populations differed significantly from each other: some studies involved large populations, ${ }^{17-19}$ and others much smaller populations with less statistical power. ${ }^{18}$ Similarly, the age of the population is another important factor in an interpretation of the results. One of the major limitations of some of the investigations was the relatively young age of their study populations. ${ }^{17-19}$ As adolescence and young adulthood are critical periods as concerns the development of MN, it appears essential to focus separately on these age groups, too.

It appears doubtful to make use of recollected anamnestic data regarding the neonatal history of the subjects. It is unacceptable simply to rely on data obtained from interviews with the parents without utilization of the official neonatal medical charts. The treatments had often occurred years or even decades before the clinical surveys, and after such a long time it is not always easy to reconstruct exactly the phototherapeutic 
modalities applied, e.g. the dose and intensity of NBLP, which can significantly influence the potential long-term side-effects.

Most studies to date have investigated the effects of NBLP on full-term infants. Certain clinical observations point to the possibility of different effects of NBLP on full-term and preterm infants; the potential minor complications of phototherapy, such as dehydration, temperature instability and electrolyte imbalance are generally fairly benign in mature neonates, but they can be much more serious in low-birthweight infants. ${ }^{57,58}$ In their multicentre, randomized trial, Morris et al. ${ }^{59}$ found an increase in mortality rate in extremely low-birthweight infants $(<750 \mathrm{~g})$ who received aggressive phototherapy. Phototherapy may significantly affect the haemodynamics of various organs, increase the cerebral and peripheral blood flow, enhance transepidermal water loss, decrease the cardiac output and the renal and mesenteric blood flows, and cause reopening of the ductus arteriosus in preterm infants by modifying the blood endothelin and nitric oxide levels. ${ }^{57,60}$ NBLP can induce a higher frequency of sister chromatid exchanges in the peripheral lymphocytes of preterm icteric neonates, ${ }^{61,62}$ and also lead to oxidative stress. ${ }^{8}$ The antioxidant defence capacity of low-birthweight, immature infants is considerably lower than that of term neonates. In preterm infants, the thickness of the stratum corneum is significantly reduced, and the production of melanin in the immature melanocytes is deficient, resulting in a limited photoprotective capacity. In view of the special characteristics of their skin, preterm infants may be particularly sensitive to intensive phototherapy, and it appears advisable to investigate the MN count of this special study population, too.

In conclusion, many of the data that are currently available on how NBLP influences naevus development are controversial. The results of recent well-designed epidemiological surveys suggest that NBLP could well be a risk factor for MN formation, and highlight the need for additional in vivo and in vitro studies. NBLP is at present the mainstay of the treatment of neonatal jaundice, and has proved to be an essential and generally safe modality for the prevention of bilirubin encephalopathy, but in the future greater consideration should be given to its long-term side-effects when phototherapy is indicated. It is relevant to emphasize the importance of appropriately restricted and adequate clinical guidelines, and strict monitoring of the management of hyperbilirubinaemia, in order to avoid the unnecessary overtreatment of newborn infants.

\section{References}

1 Moerschel SK, Cianciaruso LB, Tracy LR. A practical approach to neonatal jaundice. Am Fam Physician 2008; 77:1255-62.

2 Maisels MJ, McDonagh AF. Phototherapy for neonatal jaundice. N Engl J Med 2008; 358:920-8.

3 Stokowski LA. Fundamentals of phototherapy for neonatal jaundice. Adv Neonatal Care 2011; 11:S10-21.

4 Chen A, Du L, Xu Y et al. The effect of blue light exposure on the expression of circadian genes: bmall and cryptochrome 1 in peripheral blood mononuclear cells of jaundiced neonates. Pediatr Res 2005; 58:1180-4.
5 Csoma Z, Kemeny L, Olah J. Phototherapy for neonatal jaundice. N Engl J Med 2008; 358:2523-4.

6 Tan KL. Efficacy of fluorescent daylight, blue, and green lamps in the management of nonhemolytic hyperbilirubinemia. J Pediatr 1989; 114:132-7.

7 Liebel F, Kaur S, Ruvolo E et al. Irradiation of skin with visible light induces reactive oxygen species and matrix-degrading enzymes. J Invest Dermatol 2012; 132:1901-7.

8 Gathwala G, Sharma S. Phototherapy induces oxidative stress in premature neonates. Indian J Gastroenterol 2002; 21:153-4.

9 Tatli MM, Minnet C, Kocyigit A, Karadag A. Phototherapy increases DNA damage in lymphocytes of hyperbilirubinemic neonates. Mutat Res 2008; 654:93-5.

10 Aspberg S, Dahlquist G, Kahan T, Källén B. Confirmed association between neonatal phototherapy or neonatal icterus and risk of childhood asthma. Pediatr Allergy Immunol 2010; 21:e733-9.

11 Karadag A, Yesilyurt A, Unal S et al. A chromosomal-effect study of intensive phototherapy versus conventional phototherapy in newborns with jaundice. Mutat Res 2009; 676:17-20.

12 Messner $\mathrm{KH}$, Maisels MJ, Leure-DuPree AE. Phototoxicity to the newborn primate retina. Invest Ophthalmol Vis Sci 1978; 17:178-82.

13 Dobson V, Cowett RM, Riggs LA. Long-term effect of phototherapy on visual function. J Pediatr 1975; 86:555-9.

14 Zimmermann LE. Melanocytes, melanocytic nevi and melanomas. Invest Ophthalmol 1965; 4:14.

15 Weis E, Shah CP, Lajous M et al. The association of cutaneous and iris nevi with uveal melanoma: a meta-analysis. Ophthalmology 2009; 116:536-43.

16 Csoma Z, Tóth-Molnár E, Balogh $\mathrm{K}$ et al. Neonatal blue light phototherapy and melanocytic nevi: a twin study. Pediatrics 2011; 128:e856-64.

17 Bauer J, Büttner P, Luther $\mathrm{H}$ et al. Blue light phototherapy of neonatal jaundice does not increase the risk for melanocytic nevus development. Arch Dermatol 2004; 140:493-4.

18 Matichard E, Le Hénanff A, Sanders A et al. Effect of neonatal phototherapy on melanocytic nevus count in children. Arch Dermatol 2006; 142:1599-604.

19 Mahé E, Beauchet A, Aegerter P, Saiag P. Neonatal blue-light phototherapy does not increase nevus count in 9-year-old children. Pediatrics 2009; 123:e896-900.

20 Csoma Z, Hencz P, Orvos H et al. Neonatal blue-light phototherapy could increase the risk of dysplastic nevus development. Pediatrics 2007; 119:1036-7.

21 Oláh J, Csoma ZS, Ócsai $\mathrm{H}$ et al. Az újszülöttkori kékfény kezelés növelhet-e a felnőttkori melanoma kockázatát? Börgyógy Vener Szle 2009; 85:67-72.

22 Berg P, Lindelof B. Is phototherapy in neonates a risk factor for malignant melanoma development? Arch Pediatr Adolesc Med 1997; 151:1185-7.

23 Noell WK, Walker VS, Kang BS et al. Retinal damage by light in rats. Invest Ophthalmol 1966; 5:450-73.

24 Noell WK. Possible mechanisms of photoreceptor damage by light in mammalian eyes. Vision Res 1980; 20:1163-71.

25 Kuwabara T, Funahashi M. Light damage in the developing rat retina. Arch Ophthalmol 1976; 94:1369-74.

26 Remé CE, Grimm C, Hafezi $\mathrm{F}$ et al. Apoptosis in the retina: the silent death of vision. News Physiol Sci 2000; 15:120-4.

27 Sparrow JR, Zhou J, Ben-Shabat $S$ et al. Involvement of oxidative mechanisms in blue-light-induced damage to A2E-laden RPE. Invest Ophthalmol Vis Sci 2002; 43:1222-7.

28 Braunstein RE, Sparrow JR. A blue-blocking intraocular lens should be used in cataract surgery. Arch Ophthalmol 2005; 123:547-9.

29 Hawse P. Blocking the blue. Br J Ophthalmol 2006; 90:939-40. 
30 Manning WS Jr, Greenlee PG, Norton JN. Ocular melanoma in a Long Evans rat. Contemp Top Lab Anim Sci 2004; 43:44-6.

31 Di Cesare S, Maloney S, Fernandes BF et al. The effect of blue light exposure in an ocular melanoma animal model. J Exp Clin Cancer Res 2009; 28:48.

32 Marshall JC, Gordon KD, McCauley CS et al. The effect of blue light exposure and use of intraocular lenses on human uveal melanoma cell lines. Melanoma Res 2006; 16:537-41.

33 Algvere PV, Marshall J, Seregard S. Age-related maculopathy and the impact of blue light hazard. Acta Ophthalmol Scand 2006; 84:4 15.

34 Fielder AR, Moseley MJ. Environmental light and the preterm infant. Semin Perinatol 2000; 24:291-8.

35 Lerman S. Radiant Energy and the Eye. New York: Macmillan Publishing Co. Inc., 1980.

36 Zigman S. Lens UVA photobiology. J Ocul Pharmacol Ther 2000; 16:161-5.

37 Singh AD, Rennie IG, Seregard S et al. Sunlight exposure and pathogenesis of uveal melanoma. Surv Ophthalmol 2004; 49:41928.

38 Imesch PD, Wallow IH, Albert DM. The color of the human eye: a review of morphologic correlates and of some conditions that affect iridial pigmentation. Surv Ophthalmol 1997; 41(Suppl. 2): S117-23.

39 Bito LZ, Matheny A, Cruickshanks KJ et al. Eye color changes past early childhood. The Louisville Twin Study. Arch Ophthalmol 1997; 115:659-63.

40 Nordlund JJ, Kirkwood J, Forget BM et al. Demographic study of clinically atypical (dysplastic) nevi in patients with melanoma and comparison subjects. Cancer Res 1985; 45:1855-61.

41 Albert DM, Searl SS, Forget B et al. Uveal findings in patients with cutaneous melanoma. Am J Ophthalmol 1983; 95:474-9.

42 Tóth-Molnár E, Oláh J, Dobozy A, Hammer H. Ocular pigmented findings in patients with dysplastic naevus syndrome. Melanoma Res 2004; 14:43-7.

43 Hammer H, Tóth-Molnár E, Oláh J, Dobozy A. Cutaneous dysplastic naevi: risk factor for uveal melanoma. Lancet 1995; 346:255-6.

44 Tóth-Molnár E, Hammer H, Oláh J. Cutaneous dysplastic naevi in uveal melanoma patients: markers for prognosis? Melanoma Res 2000; 10:36-9.

45 Chin KC, Moseley MJ, Bayliss SC. Light transmission of phototherapy eyeshields. Arch Dis Child 1987; 62:970-1.

46 Moseley H, Marshall J. Protective light shields for neonatal phototherapy. Lancet 1988; 2:854-5.
47 Fok TF, Wong W, Cheung KL. Eye protection for newborns under phototherapy: comparison between a modified headbox and the conventional eyepatches. Ann Trop Paediatr 1997; 17:349-54.

48 Ostrowski G, Pye SD, Laing IA. Do phototherapy hoods really protect the neonate? Acta Paediatr 2000; 89:874-7.

49 Robinson J, Moseley MJ, Thompson JR, Fielder AR. Eyelid opening in preterm neonates. Arch Dis Child 1989; 64:943-8.

50 Moseley MJ, Bayliss SC, Fielder AR. Light transmission through the human eyelid: in vivo measurement. Ophthalmic Physiol Opt 1988; 8:229-30.

51 Robinson J, Bayliss SC, Fielder AR. Transmission of light across the adult and neonatal eyelid in vivo. Vision Res 1991; 31:1837-40.

52 Shah CP, Weis E, Lajous M et al. Intermittent and chronic ultraviolet light exposure and uveal melanoma: a meta-analysis. Ophthalmology 2005; 112:1599-607.

53 Cremer RJ, Perryman PW, Richards DH. Influence of light on the hyperbilirubinaemia of infants. Lancet 1958; 1:1094-7.

54 Maisels MJ, Kring EA, DeRidder J. Randomized controlled trial of light-emitting diode phototherapy. J Perinatol 2007; 27:565-7.

55 American Academy of Pediatrics Subcommittee on Hyperbilirubinemia. Management of hyperbilirubinemia in the newborn infant 35 or more weeks of gestation. Pediatrics 2004; 114:297-316.

56 Newman TB, Kuzniewicz MW, Liljestrand P et al. Numbers needed to treat with phototherapy according to American Academy of Pediatrics guidelines. Pediatrics 2009; 123:1352-9.

57 Benders MJ, van Bel F, van de Bor M. Haemodynamic consequences of phototherapy in term infants. Eur J Pediatr 1999; 158:323-8.

58 Jangaard KA, Vincer MJ, Allen AC. A randomized trial of aggressive versus conservative phototherapy for hyperbilirubinemia in infants weighing less than $1500 \mathrm{~g}$ : short- and long-term outcomes. Paediatr Child Health 2007; 12:853-8.

59 Morris BH, Oh W, Tyson JE et al. Aggressive vs. conservative phototherapy for infants with extremely low birth weight. N Engl J Med 2008; 359:1885-96.

60 Liu GS, Wu H, Wu BQ et al. Effect of phototherapy on blood endothelin and nitric oxide levels: clinical significance in preterm infants. World J Pediatr 2008; 4:31-5.

61 Ayyash H, Hadjigeorgiou E, Sofatzis I et al. Green or blue light phototherapy for neonates with hyperbilirubinaemia. Arch Dis Child 1987; 62:843-5.

62 Galla A, Kitsiou-Tzeli S, Gourgiotis D et al. Sister chromatid exchanges in peripheral lymphocytes in newborns treated with phototherapy and vitamin E. Acta Paediatr 1992; 81:820-3. 